\title{
Metabolitos de frutas vermelhas para um envelhecimento saudável do cérebro
}

\author{
Cláudia Nunes dos Santos*, Inês Sousa Costa", Ricardo Boavida Ferreira**, Inês Figueira"***, \\ Diana Macedo"***, Regina Menezes" ${ }^{* * * *}$, Paula Pinto ${ }^{* * * * * *}$, Lucélia Tavares
}

\section{Resumo}

O envelhecimento é considerado um processo natural de ir ficando mais velho e um reconhecido factor de risco para o desenvolvimento de doenças crónicas incluindo as doenças neurodegenerativas. A nutrição têm vindo a ser indicada como um factor importante e modificável com impacto na sáude e bem estar dos idosos. Entre os compostos bioativos da dieta destacam-se os polifenóis, que estão presents em grandes quantidades nos pequenos frutos. A procura de um envelhecimento saudável levou a uma investigação extensiva das propriedades benéficas para a saúde dos polifenóis de pequenos frutos por estudos in vitro, baseados em modelos celulares, modelos animais assim como estudos em humanos. Esta revisão discute o estado-da-arte do conhecimento sobre o impacto dos polifenóis de frutas vermelhas na saúde humana, as lacunas e a estratégia do laboratório de investigação Nutrição Molecular e Saúde para suportar a exploração dos polifenóis de pequenos frutos como moduladores de um envelhecimento saudável.

Palavras-chave: Envelhecimento Humano. Nutrição. Polifenóis. Cérebro.

* PhD em Bioquímica. Pesquisadora do Instituto de Biologia Experimental e Tecnológica e do Instituto de Tecnologia Química e Biológica, Universidade Nova de Lisboa, Av. da República, Apartado 127, 2781-901 Oeiras, Portugal. E-mail: csantos@ibet.pt

** Mestre em Bioquímica. Pesquisadora do Instituto de Biologia Experimental e Tecnológica. Pesquisadora do Instituto de Tecnologia Química e Biológica, Universidade Nova de Lisboa, Portugal.

*** PhD em Bioquímica. Pesquisador do Instituto de Tecnologia Química e Biológica, Universidade Nova de Lisboa e Professor Catedrático do Instituto Superior de Agronomia, Tapada da Ajuda 1349-017 Lisboa, Portugal. E-mail: rbferreira@isa.utl.pt

**** $\quad$ Mestre em Biologia Molecular e Genética. Doutoranda no Instituto de Tecnologia Química e Biológica, Universidade Nova de Lisboa, Portugal. E-mail: inesf@itqb.unl.pt

****** Instituto de Medicina Molecular, Faculdade de Medicina de Lisboa. Doutoranda no Instituto de Tecnologia Química e Biológica, Universidade Nova Lisboa, Portugal. E-mail: dmacedo@itqb.unl.pt

Pesquisadora do Instituto de Biologia Experimental e Tecnológica. Estagio pós-doutoral no Instituto de Tecnologia Química e Biológica, Universidade Nova de Lisboa, Portugal. E-mail: rmenezes@itqb.unl.pt Doutora em Bioquímica. Pesquisadora do Instituto de Tecnologia Química e Biológica, Universidade Nova de Lisboa e Professora na Escola Superior Agrária, Instituto Politécnico de Santarém, Portugal. E-mail: paulapinto@itqb.unl.pt

$\mathrm{PhD}$ em Bioquímica. Pesquisadora do Instituto de Biologia Experimental e Tecnológica. Estágio Pós-Doutoral no Instituto de Tecnologia Química e Biológica, Universidade Nova de Lisboa, Portugal. E-mail: ltavares@itqb.unl.pt

$\rightarrow$ http://dx.doi.org/10.5335/rbceh.v12i3.6082

Recebido em: 03/11/2015. Aceito em: 30/11/2015. 


\section{Importância da nutrição para um envelhecimento saudável}

Ao nível global, como reflexo do aumento da esperança média de vida e da diminuição da taxa de natalidade, o escalão etário das pessoas com mais de 60 anos está a aumentar, em valor relativo, mais rapidamente que qualquer outro. $\mathrm{O}$ envelhecimento populacional é um caso de sucesso das políticas de saúde pública e desenvolvimento socioeconómico, mas é também um grande desafio para a sociedade: assegurar um envelhecimento saudável.

O envelhecimento é um processo complexo, irreversível, progressivo e natural, que se caracteriza por modificações morfológicas, psicológicas, funcionais e bioquímicas afetando o bem-estar e a saúde do indivíduo. A Organização Mundial de Saúde (OMS) descreve a qualidade de vida associada ao envelhecimento como um "conceito amplo e subjetivo que integra a saúde física, o estado psicológico, o grau de independência, as relações sociais, as crenças e convicções pessoais e a sua relação com aspetos importantes do meio ambiente" (WHO, 2002, p. 12). A OMS define ainda "Envelhecimento Ativo" como o processo de otimização das oportunidades para a saúde, participação social e segurança, para melhorar a qualidade de vida das pessoas que envelhecem. Esta maximização das capacidades funcionais e saúde dos idosos é condicionada pelos fatores indicados na Figura 1 e em última análise definem o envelhecimento saudável. Alguns destes fatores não são modificáveis, como os genéticos, enquan- to outros são passíveis de modificação (ambientais, psicológicos, sociais e estilo de vida). Neste sentido a nutrição, bem como outros fatores ambientais, têm um enorme impacto na saúde e bem-estar.

Figura 1 - Fatores condicionantes de envelhecimento saudável

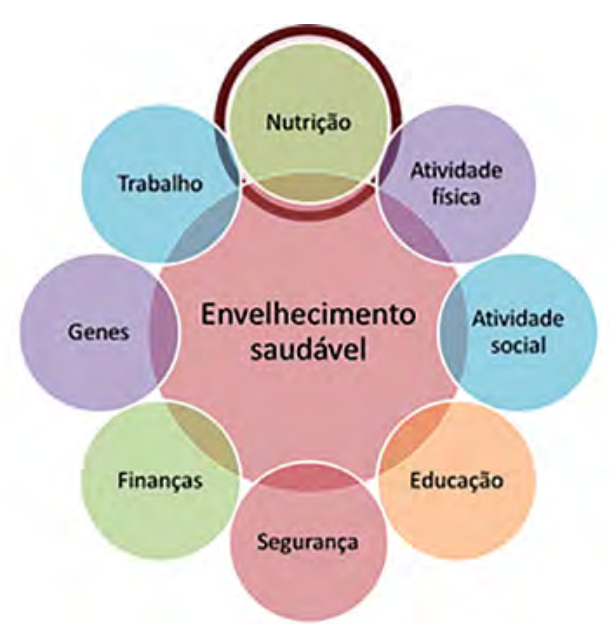

Fonte: primária.

\section{Incidência das doenças degenerativas com 0 envelhecimento}

\section{Custo e a necessidade de alternativas}

O avançar da idade está associado ao aumento da incidência de doenças degenerativas, sendo este mais acentuado a partir dos 65 anos. As patologias mais prevalentes são as doenças cardiovasculares, cancro e doenças neurodegenerativas, como as doenças de Alzheimer (DA), Parkinson (DP), Huntington (DH) e esclerose lateral amiotrófica (ELA). Este último grupo de doenças, ainda incuráveis, caracteriza-se pela perda lenta e progressiva de neurónios que 
conduz à perda de determinadas funções do sistema nervoso central (COPPEDÈ et al., 2006).

O impacto económico e social destas doenças, quer para os idosos e suas famílias, quer para os sistemas de saúde, é enorme e tende a aumentar nos anos futuros. Segundo a OMS, em 2004, as doenças neurodegenerativas afetavam 29,4 milhões de pessoas em todo o mundo (WHO, 2008), sendo a estimativa do número de pessoas com Alzheimer em 2040 de 81,1 milhões apenas na Europa (WIMO et al., 2011). O mesmo estudo aponta o custo por doente com doença neurodegenerativa de $22.000 \square$ por ano, na Europa (WIMO et al., 2011). Prevê-se então um aumento dos custos, quase insustentável para os sistemas de saúde públicos, o que torna urgente encontrar soluções. De acordo com a Alzheimer Research Trust, se a ciência desenvolver um tratamento que reduza o défice cognitivo severo em pessoas idosas em pelo menos $1 \%$ ao ano, o aumento estimado nos custos de cuidados a longo prazo devido ao envelhecimento da população seriam anulados.

\section{0 potencial dos compostos bioativos na progressão de doença}

No sentido de se procurarem soluções e considerando o progresso normal de evolução de um estado saudável para um estado de doença (Figura 2), têm emergido evidências demonstrando o papel ativo que alguns compostos presentes na dieta podem ter. Provas concretas revelam que os compostos bioativos conseguem manter as pessoas num estado de homeostasia, atrasando ou mesmo revertendo a transição de um estado saudável para um estado patológico. A nutrição, e em particular os compostos bioativos identificados na nossa dieta, surgem assim com uma forte contribuição para a manutenção de um estado salutar. Os compostos bioativos podem atuar na prevenção das doenças ou no restabelecimento do estado saudável numa fase anterior à administração de um fármaco. Este só será prescrito depois de um marcador de diagnóstico ter sido identificado e geralmente apenas trata os sintomas das doenças degenerativas, não constituindo uma cura na maior parte dos casos.

Figura 2-O desenvolvimento de doença desde o estado saudável a um estado sub-ótimo de saúde e eventualmente para estado de doença

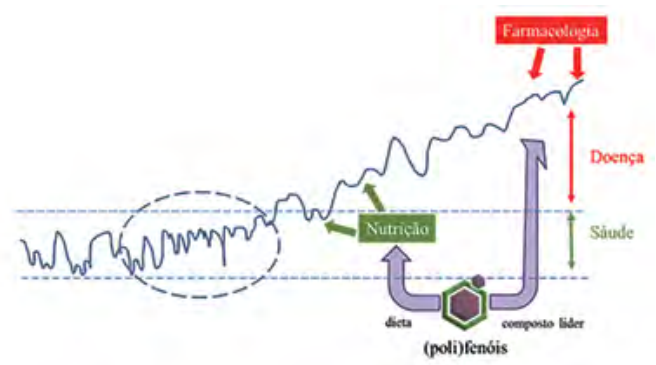

Fonte: adaptado de Van Der Greef, Stroobant e Van Der Heijden (2004).

A intervenção nutricional assume desta forma um papel muito importante na prevenção destas doenças. Estudos epidemiológicos indicam que a adesão à dieta mediterrânea, que inclui uma elevada proporção de frutos, legumes, azeite e vinho, influencia a incidência de doenças neurodegenerativas como DA e DP. Estudos verificaram que quanto 
maior a adesão, menor a probabilidade cumulativa de desenvolver um défice cognitivo ou DA e prolongando a vida dos doentes de DA (SCARMEAS et al., 2006; SCARMEAS et al., 2007; SCARMEAS et al., 2009).

\section{Definição de compostos bioativos, principais classes e distribuição na dieta}

Os componentes da dieta que têm surgido como compostos bioativos são genericamente designados de compostos fitoquímicos ou fitoprotetores. São compostos de origem vegetal que embora não sejam considerados nutrientes, pois não são fatores essenciais da dieta como as vitaminas, minerais, etc, e não provocarem síndromes de deficiência, afetam funções biológicas nos mamíferos e, consequentemente podem afetar a sua saúde. De acordo com a sua estrutura química, os compostos fitoquímicos dividem-se em várias classes como se indica na Figura 3, e a sua distribuição na dieta também está exemplificada, com compostos-tipo de cada classe.

Figura 3 - Classificação dos compostos fitoquímicos da dieta

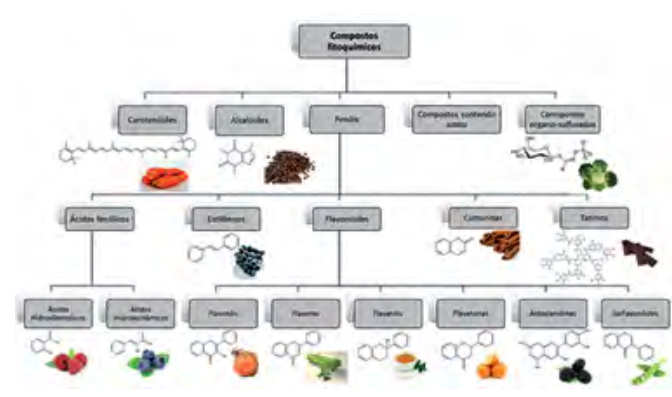

Fonte: primária.
Fenóis e saúde humana: estado atual do conhecimento, lacunas e abordagens propostas

Para se ter a real dimensão de como os fenóis, ou usualmente designados (poli)fenóis ${ }^{1}$, afetam a saúde humana e quais os mecanismos biológicos através dos quais estes compostos têm um impacto nos órgãos, é necessário conhecer (i) a ingestão de (poli)fenóis na nossa dieta, (ii) como são metabolizados, (iii) qual a sua biodisponibilidade no órgão alvo, como, por exemplo, o cérebro, e (iv) quais as suas bioatividades e os mecanismos moleculares responsáveis pela sua ação. Para cada uma destas questões identificam-se lacunas que a investigação tem tentado responder e que constituem os objetivos de investigação do laboratório da Nutrição Molecular e Sáude do Instituto de Biologia Experimental e Tecnológica/Instituto de Tecnologia Química e Biológica em Oeiras, Portugal.

\section{Consumo diário em (poli)fenóis}

A maioria dos estudos epidemiológicos observacionais que pretendem avaliar os efeitos dos (poli)fenóis na saúde assentam na estimativa do consumo diário em (poli)fenóis por um grupo populacional. Tal como apresentado na Figura 4, ao nível mundial apenas alguns países têm disponíveis estudos relativos ao consumo diário em (poli)fenóis, estando ainda muito longe de uma avaliação do consumo global. Por outro lado, muitos dos estudos analisados calculam o consumo de (poli)fenóis a partir de estudos 
epidemiológicos ou avaliações da dieta ao nível nacional efetuados há dez anos ou mais, o que poderá não representar os hábitos alimentares da população à data da publicação do estudo (ARABBI; GENOVESE; LAJOLO, 2004; CHUN; CHUNG; SONG, 2007; JOHANNOT; SOMERSET, 2006; ZAMORA-ROS et al., 2010; CASSIDY et al., 2011; PEREZ-JIMENEZ et al., 2011; TOUVIER et al., 2013; BAI; WANG; REN, 2014).

Figura 4 -Distribuição global dos estudos de avaliação do consumo de (poli)fenóis

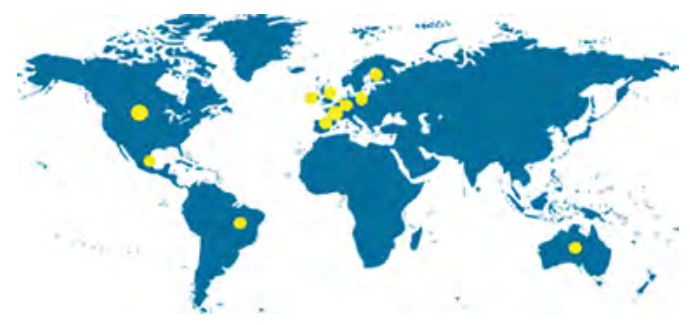

Fonte: primária.

Regista-se uma grande variabilidade dos consumos diários de (poli)fenóis reportados nos diferentes estudos, contribuindo para essas discrepâncias vários fatores: (i) Alguns estudos apenas contemplam ingestão de (poli)fenóis de frutos e/ou vegetais (ARABBI; GENOVESE; LAJOLO, 2004; PINTO et al., 2013; HERVERT-HERNANDEZ et al., 2011) excluindo importantes contribuintes como os cereais, leguminosas vinho, café, chá e cacau.

(ii) Diferentes metodologias utilizadas para avaliar o consumo alimentar (inquérito de frequência alimentar, relatório de $24 \mathrm{~h}$, questionário de histórico de dieta) que influenciam os resultados fi- nais. Alguns estudos recorreram a bases de dados de alimentos disponibilizados à população durante o ano, não refletindo de todo o consumo real da população (FAO, 2001).

(iii) Diferentes bases de dados para conversão da ingestão de alimentos em quantidade de (poli)fenóis. Três tipos de bases de dados são normalmente utilizadas: a base de dados de flavonóides e de proantocianidinas do USDA (United States Department of Agriculture) (BHAGWAT; HAYTOWITZ; HOLDEN, 2013), a base de dados de (poli)fenóis totais e de diferentes classes de (poli)fenóis do Phenol-Explorer (NEVEU et al., 2010), ou uma base de dados construída pelo próprio laboratório responsável pelo estudo a partir dos alimentos analisados.

Os valores mais elevados registados para o consumo diário em (poli)fenóis (2591 a $3016 \mathrm{mg}$ equivalente de ácido gálico (GAE)/dia) foram observados em estudos que quantificaram os (poli) fenóis extraíveis e não extraíveis no próprio laboratório (SAURA-CALIXTO; SERRANO; GOÑI, 2007), enfatizando a importância desta última fração para o cálculo do consumo total de (poli)fenóis. Os estudos que recorrem à base de dados Polyphenol-Explorer, a qual apenas contabiliza os (poli)fenóis extraíveis, apresentam valores entre os 564 e os $1781 \mathrm{mg}$ GAE/dia (OVASKAINEN et al., 2008; PEREZ-JIMENEZ et al., 2011; TRESSERRA-RIMBAU et al., 2013; TOUVIER et al., 2013; GROSSO et al., 2014).

Por outro lado, estudos que utilizam a base de dados do USDA contabilizam apenas os flavonóides e as proanto- 
cianidinas apresentando valores mais baixos, entre 313 a $385 \mathrm{mg}$ GAE/dia (JOHANNOT; SOMERSET, 2006; CHUN; CHUNG; SONG, 2007; CASSIDY et al., 2011; BEKING; VIEIRA, 2011; BAI; WANG; REN, 2014), deixando de fora os ácidos fenólicos, uma importante classe de (poli)fenóis que contribui com 37 a $74 \%$ do consumo total diário (OVASKAINEN et al., 2008; TRESSERRA-RIMBAU et al., 2013; PEREZ-JIMENEZ et al., 2011; GROSSO et al., 2014).

Tendo em conta o consumo de (poli) fenóis não extraíveis, o grupo de alimentos que mais contribui para o consumo de (poli)fenóis é o grupo dos cereais devido ao seu elevado conteúdo em (poli) fenóis hidrolisáveis (SAURA-CALIXTO; SERRANO; GOÑI, 2007). Considerando apenas os (poli)fenóis extraíveis, as bebidas como o café e o chá são os alimentos que mais contribuem para o consumo de (poli)fenóis. A contribuição dos restantes grupos de alimentos como frutos, vegetais, vinho e cacau, dependem grandemente dos hábitos alimentares do país. Por exemplo os frutos podem contribuir com 5.6\% na Finlândia (OVASKAINEN et al., 2008) até $44 \%$ em Espanha (TRESSERRA-RIMBAU et al., 2013).

Num estudo realizado em Portugal, foi determinado o consumo de (poli)fenóis proveniente de frutos frescos e em particular dos pequenos frutos, alimentos muito ricos em (poli)fenóis (PINTO et al., 2013). O consumo total diário de frutos foi de $365.68 \mathrm{~g} / \mathrm{dia}$, dos quais $9 \%$ foram de pequenos frutos, principalmente morangos. No entanto, a contribuição dos pequenos frutos para o consumo total de (poli)fenóis é bastante significativa comparativamente com outros frutos, contribuindo com $14 \%$ do valor total de (poli)fenóis consumidos (783.93 mg GAE/dia).

\section{Metabolismo e eliminação}

Para melhor compreender como atuam os (poli)fenóis no corpo humano é essencial compreender a sua biodisponibilidade. Depois da ingestão dos alimentos, os compostos sofrem diversas transformações no trato gastrointestinal, bem como depois da sua absorção para a corrente sanguínea. Para além disso, uma grande quantidade de compostos que não são absorvidos no intestino delgado chegam ao cólon onde são catabolizados pelo microbiota aí presente, originando fenóis mais simples que ainda poderão ser absorvidos e metabolizados. No entanto, estes eventos não estão totalmente elucidados, tal como não são ainda conhecidos a maioria dos metabolitos originados durante a digestão a partir dos (poli)fenóis.

Os (poli)fenóis que ingerimos com mais frequência não são necessariamente os mais ativos no organismo humano. Isto prende-se com o fato de os diferentes compostos apresentarem diferentes atividades e serem diferencialmente absorvidos, metabolizados e excretados. Absorção, metabolismo e excreção são assim passos essenciais para se compreender o efeito dos (poli)fenóis na saúde humana e consequentemente estratégias para potenciar os seus efeitos.

Durante a digestão e metabolismo os (poli)fenóis sofrem várias alterações estruturais, resultando em metabolitos que circulam no sangue e atingem te- 
cidos, mas que diferem dos compostos nativos quanto à sua estrutura química e atividade biológica. Existem vários passos cruciais neste processo, onde a metabolização no trato gastrointestinal e absorção na mucosa que funciona como uma barreira física são as primeiras etapas. Sendo lipofílicos, a maior parte das aglíconas de flavonoides são permeáveis nas células intestinais e difundem-se passivamente. No entanto, nas plantas, os (poli)fenóis estão normalmente presentes sob a forma de ésteres, glucósidos e polímeros.

Todas estas formas não são absorvidas diretamente e provavelmente resistem a hidrólise ácida no estômago, podendo alcançar o duodeno. Para os compostos glicosilados, a absorção é usualmente precedida pela separação do açúcar da aglícona. Em alguns compostos esta reação ocorre no intestino delgado, no entanto alguns glucósidos não são hidrolisados nesta fase seguindo para o cólon onde são metabolizados pelo microbiota e posteriormente absorvidos. O microbiota do cólon pode promover várias transformações na estrutura dos (poli)fenóis incluindo O- e C-deglicosilação, hidrólise de ésteres e amidas e desglucoronidação. Após absorção, os metabolitos originados a partir dos (poli)fenóis são ainda conjugados, via reações de fase I e fase II que ocorrem principalmente no fígado. O passo final é a eliminação e/ou destoxificação através da excreção (Figura 5).
Figura 5 - Diagrama geral de absorção, biotransformação e excreção dos (poli) fenóis no organismo humano

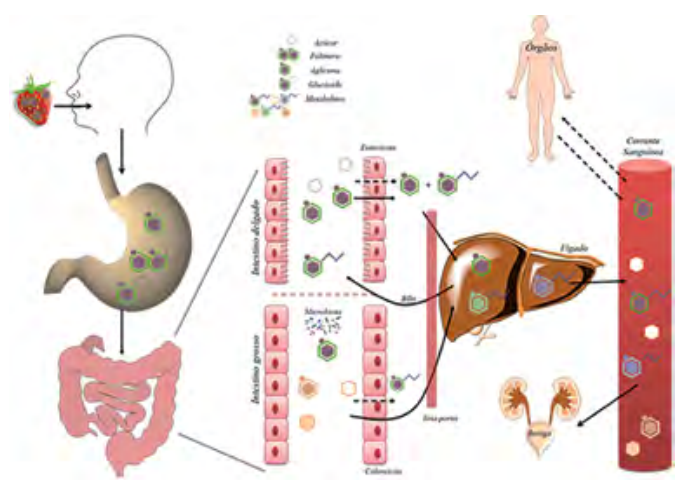

Fonte: Santos et al. (2015).

Estudo de intervenção em humanos com puré de pequenos frutos

Os pequenos frutos têm um reconhecido elevado valor nutricional e estão descritos com vários efeitos benéficos para a saúde (PAREDES-LOPEZ et al., 2010; BASU; LYONS, 2011; KOLEHMAINEN et al., 2012). Com o intuito de identificar os metabolitos biodisponíveis mais abundantes após a ingestão de (poli)fenóis de um puré de pequenos frutos e prever a sua importância para a saúde humana, o laboratório de Nutrição Molecular e Sáude realizou um estudo de intervenção com humanos. Preparou-se uma mistura de pequenos frutos, constituída por mirtilos, amoras e framboesas comerciais e ainda dois frutos selvagens, o medronho e a camarinha. Esta mistura de frutos apresentou uma elevada diversidade química de (poli)fenóis (PIMPÃO et al., 2013). Após ingestão da mistura pelos voluntários, foram colhidas amostras de sangue e urina em vários pontos temporais (Figura 6A). 
Com base na literatura existente, foi construída uma biblioteca in silico dos potenciais metabolitos biodisponíveis e esta foi usada como referência para a análise exploratória das amostras de urina, efetuada por cromatografia líquida acoplada a um espectrómetro de massas exatas (Orbitrap). A confirmação da identificação dos metabolitos foi efetuada por LC-MS/MS através da comparação com compostos padrão sintetizados química ou enzimaticamente. Vários metabolitos foram identificados nas amostras de urina, maioritariamente na forma conjugada com grupos metilo, ácido glucurónico e sulfato, sendo que alguns foram identificados em amostras humanas pela primeira vez (PIMPÃO et al., 2014).

Posteriormente, estes compostos foram quantificados no plasma e a sua origem a partir dos (poli)fenóis ingeridos foi confirmada através de um estudo de intervenção cruzado (Figura 6B), em que os voluntários humanos ingeriram um puré de pequenos frutos numa fase e na outra um pequeno-almoço livre de (poli) fenóis (PIMPÃO et al., 2015).

No entanto, o número crescente de estudos que evidenciam a biodisponibilidade na circulação sanguínea dos (poli)fenóis e dos seus metabolitos (MANACH et al., 2004; MANACH et al., 2005; WILLIAMSON; MANACH, 2005; PIMPÃO et al., 2014) não colmata ainda a reduzida informação disponível acerca da sua capacidade para alcançar órgãos-alvo, e aí, exercer os seus efeitos. Em particular, é muito importante entender a permeabilidade de alguns órgãos, considerados santuários farmacológicos, como o cérebro, placenta e testículos a estes metabolitos circulantes, os verdadeiros compostos com potencialidade para efeitos biológicos.

Figura 6 - Representação do estudo de intervenção em humanos

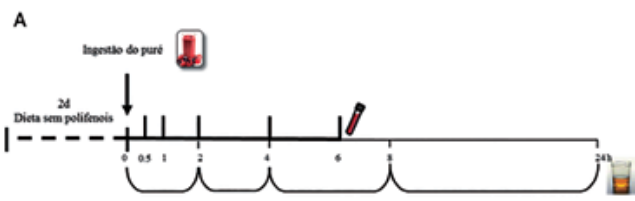

B

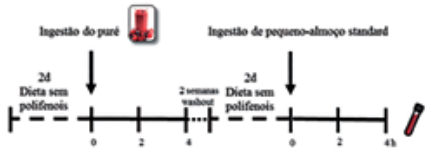

Nota: A. Estudo cinético de biodisponibilidade com os tempos de colheitas de sangue e intervalos de tempos de colheita de urina indicados. B. Estudo cruzado com os tempos de colheita de sangue indicados.

Fonte: primária.

\section{Estudos das bioatividades para o cérebro}

Atualmente, existem várias evidências científicas do benefício da ingestão de pequenos frutos para a memória e manutenção do estado cognitivo, o qual é deteriorado com o envelhecimento (SHUKITT-HALE; LAU; JOSEPH, 2008; MALIN et al., 2011). Em modelos animais, usando ratos envelhecidos, o consumo de amoras melhorou a performance em testes motores relacionados com equilíbrio e coordenação motora, bem como em testes que avaliaram a memória espacial (SHUKITT-HALE; CHENG; JOSEPH, 2009).

À luz do conhecimento atual, propõem-se mecanismos de ação dos (poli) fenóis muito para além das suas propriedades como sequestradores de radicais livres (antioxidantes): pensa-se que os 
(poli)fenóis possam exercer os seus efeitos no cérebro por vias indiretas, através da ativação de mecanismos de hormese e através de efeitos nos sistemas periféricos do corpo humano, que, em última instância, influenciam o funcionamento do sistema nervoso central (SCHAFFER; HALLIWELL, 2012). A hormese descreve o mecanismo pelo qual a exposição a doses baixas de um metabolito, que poderá ser tóxico em doses mais elevadas, é capaz de desencadear efeitos benéficos nas células ou no organismo (MATTSON; CHENG, 2006).

Neste sentido, é importante ajustar o estudo dos (poli)fenóis para além do dogma reducionista (poli)fenol-efeito: é preciso abranger não só os (poli)fenóis, como também os metabolitos resultantes da sua digestão em concentrações que, apesar de baixas, sejam fisiologicamente relevantes, tendo em conta a multiplicidade de ações entre diferentes órgãos do corpo humano num benefício global para o sistema nervoso central.

A abordagem mais utilizada atualmente contempla a avaliação in vitro dos (poli)fenóis em condições de ensaio próximas das condições fisiológicas, em modelos celulares humanos de doença. Não utilizando os (poli)fenóis isolados mas recorrendo alternativamente aos seus metabolitos biodisponíveis, quando conhecidos, ou ainda recorrendo a modelos in vitro de digestão gastrointestinal, podem ser testados os metabolitos assim obtidos em células humanas em concentrações passíveis de serem encontradas no plasma sanguíneo (0-4 $\mu \mathrm{M})$ (TAVARES et al., 2012; TAVARES et al., 2013) (Figura 7). Para se estudar o efeito de amoras em células neuronais humanas, no laboratório de Nutrição Molecular e Sáude recorreu-se à digestão in vitro segundo a abordagem fisiológica proposta.

Figura 7 - Representação esquemática das diferenças entre a abordagem não fisiológica frequentemente adotada em modelos in vitro em comparação com a abordagem fisiológica recentemente adotada

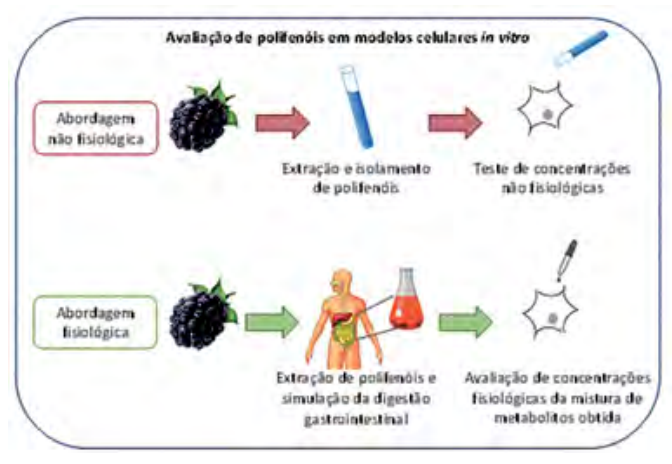

Fonte: primária.

Permeabilidade e transporte na barreira

hematoencefálica

De forma a validar a abordagem anteriormente proposta como fisiológica é também essencial assegurar, pelo menos numa pequena extensão, que os metabolitos dos (poli)fenóis conseguem atravessar a barreira hematoencefálica ou interagir com as células que a constituem e desencadear cascatas de sinalização que possam ativar processos no sistema nervoso central.

A barreira hematoencefálica é essencialmente constituída por células endoteliais, perícitos e astrócitos, formando uma camada semipermeável que exerce um controlo altamente regulado no tráfego molecular transendotelial (Figura 
8A). Os metabolitos poderão conseguir atravessar esta barreira através de mecanismos de difusão passiva (compostos lipídicos, de pequenas dimensões, transporte dependente de gradiente), por transporte ativo (mediado por transportadores de influxo ou efluxo específicos), ou ainda um transporte mediado por componentes especializados (mecanismos de endocitose, responsáveis pelo transporte de moléculas de maiores dimensões e proteínas) (FARIA; MATEUS; CALHAU, 2012). Esta regulação estrita do transporte por diversos mecanismos assegura a integridade e homeostasia das células do cérebro, permitindo a passagem de nutrientes, vitaminas e alguns fármacos, mas também limitando a passagem de substâncias potencialmente nocivas para o cérebro.

Estudos in vivo com animais (TALAVÉRA et al., 2005; EL MOHSEN et al., 2006) demonstraram que determinados (poli)fenóis são capazes de atravessar a barreira hematoencefálica independentemente da via de administração, tendo sido detetados em tecidos cerebrais. Os (poli)fenóis apresentam-se então como potenciais candidatos a exercerem ações diretas neuroprotetoras e neuromoduladoras. Contudo, os metabolitos biodisponíveis dos (poli)fenóis deverão ser transportados em concentrações que lhes permitam atuar sobre os seus alvos, dependendo intimamente da sua absorção, metabolismo, distribuição nos tecidos, acumulação intracelular e excreção. Nesse sentido, o trabalho desenvolvido pelo laboratório de Nutrição Molecular e Sáude tenta colmatar essas limitações ainda existentes na investigação, nomeadamente acerca da permeabilidade dos (poli)fenóis na barreira hematoencefálica.

Recorrendo a um modelo simplificado da barreira hematoencefálica, constituído por células humanas endoteliais imortalizadas (STINS; BADGER; SIK, 2001; PALMELA et al., 2011), avaliou-se o transporte de metabolitos de (poli) fenóis através destas células (Figura 8B). As células cresceram em suportes especializados, permitindo a recolha dos meios superior e inferior (apical, equivalente à corrente sanguínea, e basolateral, equivalente ao parênquima cerebral, respetivamente). Os metabolitos dos (poli)fenóis foram administrados às células em concentrações fisiológicas, e foi observado o seu transporte, observando-se percentagens de transporte que vão desde 2 a $20 \%$ consoante os compostos em análise.

Por outro lado também registamos efeitos protetores diferenciais contra insultos oxidantes. Foi também avaliado este transporte ao longo do tempo e quando co-incubado com inibidores dos diferentes transportadores de efluxo, de modo a melhor elucidar acerca dos mecanismos de transporte dos metabolitos dos (poli)fenóis através da barreira hematoencefálica. Parâmetros como resistência elétrica transendotelial e permeabilidade paracelular à fluoresceína de sódio (PALMELA et al., 2011) foram também monitorizados de modo a assegurar a integridade celular da barreira durante as experiências. 
Figura 8-A. Representação esquemática da barreira hematoencefálica e dos diferentes mecanismos de transporte mediado pelas células que a constituem; B. Representação esquemática da abordagem de análise do transporte dos metabolitos de (poli) fenóis na barreira hematoencefálica

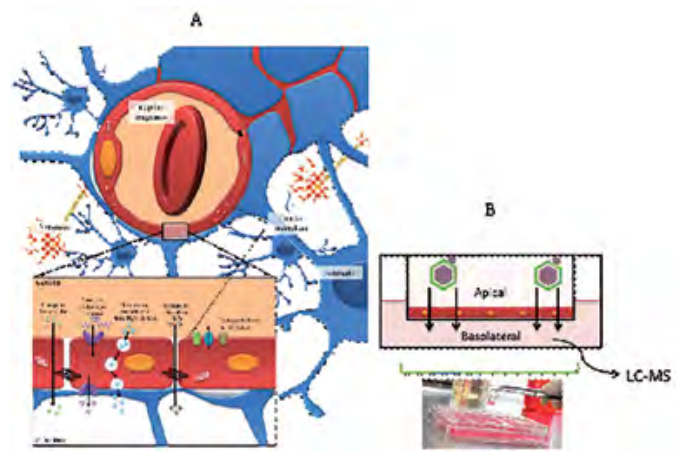

Fonte: adaptado de Figueira et al. (2016, no prelo).

Neuroprotecção num modelo celular de neurodegeneração humano e validação em células primárias de ratinho

Após verificada a possibilidade dos metabolitos dos (poli)fenóis entrarem no cérebro e decorrente das inúmeras características já mencionadas, a capacidade neuroprotetora e os mecanismos de proteção dos (poli)fenóis presentes em amoras foram avaliados num modelo celular in vitro. A metodologia utilizada teve em conta a abordagem fisiológica anteriormente explicada (Figura 7). Uma vez que o stresse oxidativo tem sido apontado como estando implicado na patogénese de diversas doenças neurodegenerativas e no envelhecimento em geral, foi utilizado um modelo celular de neurodegeneração, estabelecido a partir de uma linha celular derivada de neuroblastoma humano, submetidas a um stress oxidativo (TAVARES et al., 2013) (Figura 9).

Os resultados obtidos mostraram que os (poli)fenóis de amora resultantes da digestão protegem os neurónios de um insulto oxidativo, avaliado pela viabilidade celular (TAVARES et al., 2012). No entanto, os mecanismos protetores não resultaram da captação direta de radicais livres (atividade antioxidante direta), nem da modulação dos níveis de glutationo (principal antioxidante endógeno não enzimático) (TAVARES et al., 2012). Foi, no entanto, observada uma ativação de caspases 3 e 7 , que poderá estar relacionada com a ativação de mecanismos de pré-condicionamento e consequente proteção celular (TAVARES et al., 2013). $\mathrm{O}$ efeito protetor poder-se-á dever ainda à modulação de outros sistemas antioxidantes endógenos como enzimas antioxidantes (superóxido dismutase, catalase, etc) (TAVARES et al., 2012).

Figura 9 - Representação esquemática da abordagem de análise da capacidade neuroprotetora dos metabolitos de (poli)fenóis em células humanas

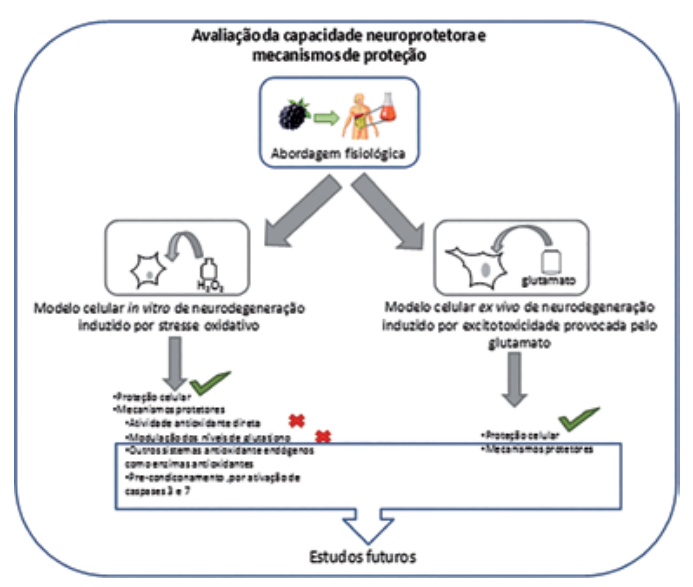

Fonte: primária. 
Com o objetivo de confirmar/validar os resultados de neuroproteção obtidos previamente em linha celular, foi desenvolvido um ensaio de neuroproteção num modelo de neurodegeneração ex vivo, desenvolvido a partir de células cerebelares granulares extraídas de ratinhos. Neste estudo, a morte celular foi induzida pela aplicação de glutamato (MEHTA et al., 2013). O glutamato é o neurotransmissor endógeno mais predominante no cérebro humano; no entanto, quando este atinge elevados níveis extracelulares é desencadeado um mecanismo de excitotoxicidade que conduz à morte neuronal. Neste último modelo foi observado que o contacto das células cerebelares granulares com metabolitos de (poli)fenóis as protegeu de forma significativa, quando comparadas com células expostas apenas ao estímulo tóxico (glutamato).

Desta forma, foi avaliada como potencialmente benéfica a ingestão de amoras na prevenção de lesões cerebrais decorrentes de um elevado stresse oxidativo ou de excitotoxicidade promovida pelo glutamato, a que os neurónios possam ser expostos.

\section{Desafios para a Indústria alimentar}

Os estudos desenvolvidos representam uma oportunidade para a indústria alimentar no sentido de utilizar os pequenos frutos no desenvolvimento de produtos alimentares que promovam um envelhecimento saudável. Estes frutos podem incorporar ingredientes funcionais, ou constituírem nutracêuticos. $\mathrm{O}$ real conhecimento das suas propriedades e formas de ação pode ajudar na definição de dietas personalizadas. Por outro lado, estudos realizados no âmbito do projeto Europeu EUBerry revelaram algumas espécies de amoras selvagens e linhas de melhoramento de framboesas e morangos com maior potencialidade para serem comercializadas com base na sua mais-valia nutracêutica. Noutra perspectiva, a caracterização das bioatividades entre a biodiversidade mundial dos pequenos frutos está a permitir identificar, no âmbito do projeto europeu BacHBerry, quais os compostos fenólicos com maior bioatividade de forma a serem produzidos em larga-escala para a indústria.

\section{Projeto EUBerry}

A ocorrência em Portugal de espécies de amoras silvestres, em estado selvagem, constitui uma enorme fonte de diversidade de compostos fitoquímicos, nomeadamente (poli)fenóis. Das espécies de amoras silvestres descritas na Península Ibérica ascende a uma dezena as identificadas em Portugal (JENNINGS, 1988). Estas espécies são diferentes das que geralmente ocorrem no resto do mundo e das que são utilizadas nos processos de melhoramento, que dão origem aos frutos que encontramos disponíveis no mercado (JENNINGS, 1988; FINN, 2008). Estas espécies de amoras pensa-se serem espécies pré-glaciares que sobreviveram às duas últimas glaciações em zonas consideradas refúgio, como a zona de Trás-os-Montes em Portugal (JENNINGS, 1988). A avaliação das espécies silvestres foi iniciada com a prospeção em campo na zona de Trás-os-Montes. A diversidade química existente nestas espécies torna a avaliação do seu 
potencial neuroprotetor extremamente interessante (TAVARES et al., 2013).

\section{Impacto para a nutrição humana}

Os (poli)fenóis das espécies de amora silvestres ( $R$. brigantinus e $R$. vagabun$d u s$ ) após digestão gastrointestinal mostraram uma clara proteção da viabilidade e funcionalidade celular no modelo testado, melhor que a obtida por amoras comerciais (TAVARES et al., 2013). Os resultados obtidos mostraram uma clara neuroprotecção mediada por mecanismos moleculares adaptativos desencadeados pelas amoras silvestres, também conhecidos como pré-condicionamento ou hormese, já referidos (TAVARES et al., 2013).

Os resultados obtidos neste trabalho evidenciam o consumo de amoras silvestres como uma mais-valia para a saúde. De forma a tirar partido deste potencial nutricional o consumo de pequenos frutos deverá ser impulsionado, bem como a disponibilização de novas espécies com reconhecidas vantagens nutricionais. Assim, espera-se que o consumidor possa vir a adquirir frutos/nutracêuticos com um elevado valor nutricional validado cientificamente em modelos celulares, sabendo que este constitui uma mais-valia na prevenção de futuras doenças neurodegenerativas.

As amoras silvestres poderão assim surgir como o próximo superfruto a chegar ao mercado e cativar a atenção do consumidor. Produtos inovadores derivados das amoras poderão ser elaborados já que a sua integridade nutricional e nutracêutica é assegurada. A introdução no mercado de produtos de valor nutricional acrescenta- do, em particular para um escalão etário da população cada vez mais significativo, como a população idosa, poderá ajudar num envelhecimento saudável.

\section{Do campo, para o laboratório e para 0 mercado}

Uma vez identificado o potencial interesse de (poli)fenóis de amoras silvestres para a saúde, é imperativo caracterizar agronomicamente estas espécies e até mesmo introduzir este germoplasma em programas de melhoramento para posterior exploração comercial. Nesse âmbito, no projeto europeu EUBerry foram efetuados ensaios agronómicos, de qualidade pós-colheita e avaliação de compostos fitoquímicos, com o objetivo de caracterizar algumas destas espécies presentes em território Português (Figura 10).

Figura 10 - Evolução dos estudos das amoras silvestres da zona envolvente de Trás-os-Montes. Da recolha no campo, para o laboratório para análise química e análise in vitro de bioatividades em modelos celulares e finalmente introdução em cultura para o mercado

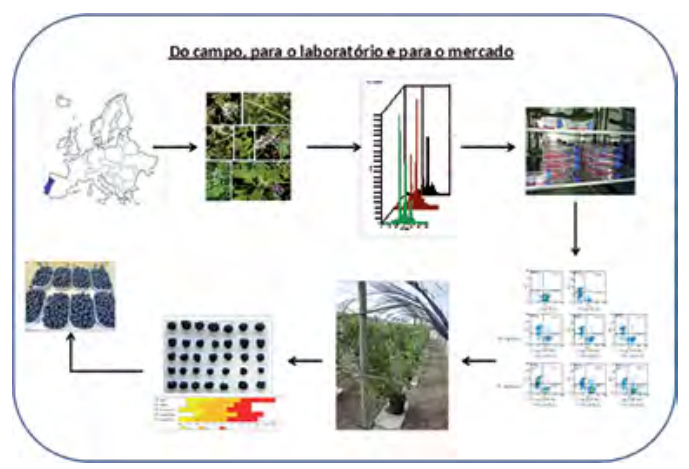

Fonte: primária.

RBCEH, Passo Fundo, v. I2, n. 3, p. 268-287, set./dez. 2015 
Nesse estudo pretendeu-se testar a introdução das espécies silvestres $R$. vigoi; $R$. radula; $R$. henriquesii; $R$. sampaioanus e $R$. brigantinus em cultura (condições favoráveis a um melhor desenvolvimento vegetativo e produtivo). Tentou-se deste modo responder às questões relacionadas com a adaptação destas espécies ao local e ao sistema de produção, quais as características vegetativas e produtivas das espécies (quantidade e qualidade da produção) e, por fim, a sua sustentabilidade económica, uma vez que podem assumir um elevado valor acrescentado na comercialização, em seccionamentos de mercado.

As espécies testadas revelaram-se bem adaptadas às condições climáticas da sua região de origem, podendo condicionar o seu cultivo em regiões do Sul ou junto ao Litoral. Concluiu-se que apenas a $R$. henriquesii e $R$. sampaioanus apresentaram características vegetativas e produtivas aceitáveis. Um melhoramento adequado poderá ainda obter variedades de amoras bem adaptadas ao clima Português.

Estas espécies apresentam-se bem adaptadas a subsistirem em condições de baixos inputs. A sua introdução em cultura permitirá: i) a sua valorização e consequente proteção das espécies; ii) potencial adaptação a sistemas de cultivo com baixos inputs, um objetivo essencial numa agricultura sustentável de futuro.

O melhoramento varietal poderá explorar a elevada variabilidade de germoplasma em território Português, mas preservando ao máximo as qualidades silvestres por forma a lhes conferir um valor acrescentado na comercialização e seccionamento de mercado.

\section{Projeto BacHBberry}

No sentido de uma produção sustentável de (poli)fenóis provenientes de pequenos frutos silvestres decorre o projeto europeu BacHBerry. Neste contexto, o BacHBerry é um projeto ambicioso, cujos principais objetivos são: (i) a bioprospecção de coleções mundiais de espécies de pequenos frutos para identificação de compostos fenólicos bioativos com potencial efeito terapêutico associado às doenças crónicas (Figura 11); (ii) a elucidação das vias biossintéticas destes compostos de forma a permitir, através da engenharia metabólica de bactérias hospedeiras, a sua produção em processos de fermentação escaláveis de última geração, e que, em última análise, sirvam como plataformas de produção comercial (Figura 11).

Figura 11 - Representação esquemática das diversas etapas do projeto $\mathrm{BacH}$ Berry

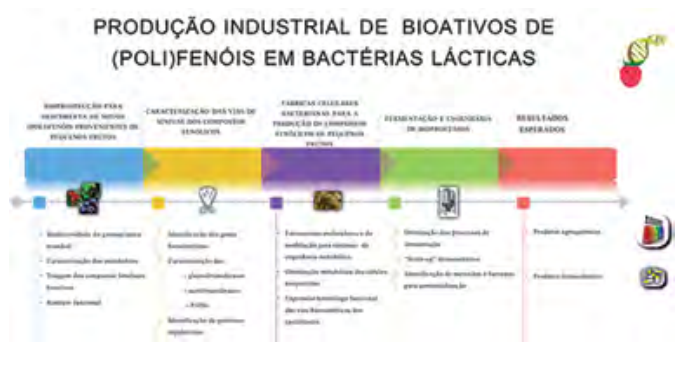

Fonte: primária.

O laboratório de Nutrição Molecular tem uma participação chave no consórcio BacHBerry, não só através da disponibilização de espécies endémicas de frutos silvestres, contribuindo para a coleção 
de germoplasma mundial, mas também mediante a bioprospecção de compostos fenólicos nos extratos de pequenos frutos enriquecidos em (poli)fenóis e nas frações selecionadas e, por último, através da avaliação das bioatividades dos compostos produzidos sinteticamente pelas bactérias.

A utilização de uma plataforma de leveduras "humanizadas", ou seja, de leveduras recombinantes que expressam genes humanos, na primeira fase de bioprospecção sistemática dos compostos fenólicos, é um dos vários aspetos inovadores deste projeto. Este sistema permite uma triagem rápida e eficaz dos extratos/ frações de compostos ativos, com capacidade de suprimir in vivo o efeito deletério da expressão de genes humanos ou ortólogos associados a doenças crónicas, tais como as doenças neurodegenerativas (DP, DA, DH e ELA), a diabetes, o cancro e a inflamação. Finalmente, as bioatividades dos principais compostos identificados e produzidos no decorrer do projeto serão validadas em modelos mais complexos de linhas celulares de mamíferos.

\section{Desafios para a Indústria farmacêutica-case-study para doença de Parkinson (DP)}

A DP é a doença neurodegenerativa motora mais comum (VON CAMPENHAUSEN et al., 2005). A prevalência de DP aumenta com a idade, tendo como idade de incidência aproximadamente 65 anos (VON CAMPENHAUSEN et al., 2005). Estima-se que o número de pacientes de DP aumente de 4,1 milhões em 2005 para 8,7 milhões em 2030 (RAO; HOFMANN; SHAKIL, 2006). A DP é caracterizada molecularmente pela perda de neurónios dopaminérgicos na substantia nigra, responsável pelos sintomas motores e psicológicos (SPILLANTINI; GOEDERT, 2001; ERIKSEN; WSZOLEK; PETRUCELLI, 2005). Estes neurónios apresentam inclusões citoplasmáticas, designadas por corpos de Lewy, constituídas principalmente por alfa-sinucleína (aSin) malconformada e agregada (ERIKSEN; WSZOLEK; PETRUCELLI, 2005; SPILLANTINI; GOEDERT, 2001). O stresse oxidativo está fortemente associado à agregação de aSin e à morte celular dos neurónios dopaminérgicos (SOUZA et al., 2000).

Apesar do extenso conhecimento molecular da DP, a farmacoterapia usada atualmente foca-se apenas na componente sintomática da doença, tentando repor os níveis de dopamina no cérebro (TARAZI et al., 2014; HORNYKIEWICZ, 2002). No entanto, têm limitada eficácia, não impedem a progressão da doença e apresentam efeitos colaterais motores e não-motores (JANKOVIC, 2005; ISACSON et al., 2008), surgindo assim uma necessidade urgente de desenvolver novas terapias. Os (poli)fenóis têm suscitado grande interesse nas doenças neurodegenerativas (SIMONYI et al., 2005; SCALBERT et al., 2005; TAVARES et al., 2012; MECOCCI et al., 2015).

(Poli)fenóis extraídos de folhas de Corema album, uma espécie endémica de Península Ibérica, revelaram grande potencial na proteção contra a DP em modelos celulares (MACEDO et al., 2015). Estes (poli)fenóis reduziram a to- 
xicidade e agregação da aSin em modelos de levedura e células humanas H4. Relativamente ao mecanismo de proteção subjacente, estes compostos reduziram a fibrilizacão da aSin in vitro; reduziram os níveis do radical superóxido, principalmente produzido pela mitocôndria em situações de stresse; e promoveram o fluxo da autofagia, a principal via de degradação dos agregados de aSin. Os mecanismos de ação estudados estão esquematizados na Figura 12 (MACEDO et al., 2015). Neste estudo foi revelado o potencial neuroprotetor da folha $C$. album na DP.

Figura 12 - Esquema dos mecanismos de protecção dos (poli)fenóis contra a toxicidade da aSin em modelos celulares

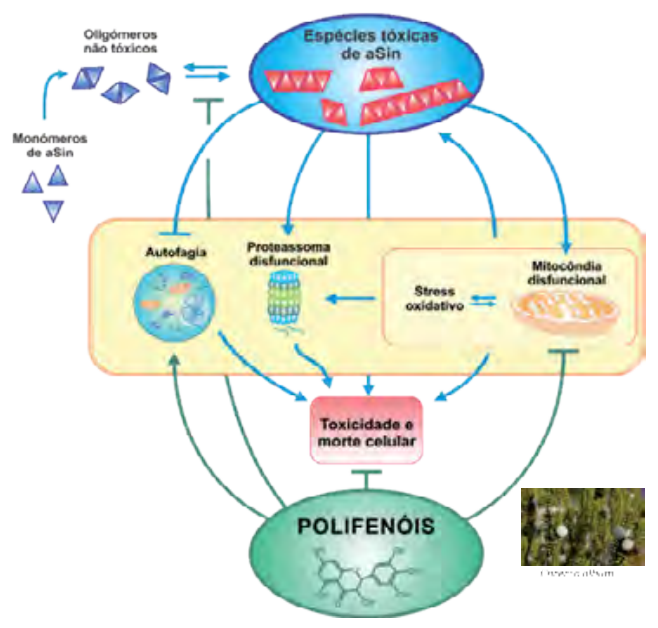

Fonte: adaptado de Macedo et al. (2015).

\section{Conclusão}

Apesar do forte potencial neuroprotetor dos nutracêuticos em modelos celulares, dos estudos de intervenção e ensaios clínicos em seres humanos (DEFEUDIS; DRIEU, 2000; MANDEL; YOUDIM, 2004; CARCACHE-BLANCO et al., 2006; AFKIR et al., 2008; MECOCCI et al., 2015), não existem diretrizes para o tratamento de doenças neurodegenerativas com nutracêuticos. Para o completo desenvolvimento do potencial dos nutracêuticos é importante que a indústria farmacêutica promova estudos clínicos sobre a sua ingestão a curto e longo prazo, focando na mitigação da gravidade e da incidência de doenças do envelhecimento, dando uma nova oportunidade para o desenvolvimento de terapias eficazes como é o exemplo apresentado contra a DP.

\section{Agradecimentos}

Os trabalhos foram suportados pela Fundação para a Ciência e a Tecnologia (PEst-OE/EQB/LA0004/2011 e bolsas individuais) e pela Comissão Europeia ( FP7 EUBerry KBBE-2010-4 265942 e FP7 BacHBerry KBBE-2013-613793). Agradece-se Alexandra Brito e Dora Brites do iMED da Faculdade de Farmácia de Lisboa pela colaboração nos trabalhos relacionados com a barreira hematoencefálica, Sandra Tenreiro e Tiago Outeiro do Instituto de Medicina Molecular de Lisboa nos trabalhos relacionados com os modelos de Parkinson, Pedro Oliveira do INIAV no estudo das características agronómicas das amoras silvestres. 


\section{Metabolites of berries for healthy aging of the brain}

\section{Abstract}

Ageing is considered a natural process of becoming older and a recognized risk factor for the development of chronic disorders including neurodegenerative diseases. Nutrition has been pointed as an important modifiable factor impacting health and well being of elderly. Among bioactive dietary compounds stands out the (poly)phenols, which are present in high amounts in berries. The quest for a healthy ageing has led to the extensive investigation of the health-promoting properties of berry (poly) phenols by means of studies using in vitro, cell-based, animal models as well as human studies. This review discusses the state-of-art knowledge on the impact of berry (poly)phenols in human health, the gaps in the body of knowledge and the strategy used by the Molecular Nutrition \& Health laboratory to support the exploitation of berry (poly)phenols as modulators of a healthy ageing.

Keywords: Healthy Aging. Nutrition. Polyphenols. Brain.

\section{Nota}

1 O termo genérico de (poli)fenóis inclui todas as subclasses do fenóis indicadas na Figura 3, incluindo os ácidos fenólicos que contêm apenas um anel fenólico.

\section{Referências}

AFKIR, S. et al. Arbutus Unedo prevents cardiovascular and morphological alterations in L-Name-Induced hypertensive rats part I: Cardiovascular and renal hemodynamic effects of Arbutus Unedo in L-Name-Induced hypertensive rats. Journal of Ethnopharmacology, v. 116, n. 2, p. 288-295, 2008.
ARABBI, P. R.; GENOVESE, M. I. S.; LAJOLO, F. M. Flavonoids in vegetable foods commonly consumed in Brazil and estimated ingestion by the Brazilian population. Journal of Agricultural and Food Chemistry, v. 52, n. 5, p. 1124-1131, 2004.

BAI, W.; WANG, C.; REN, C. Intakes of total and individual flavonoids in US adults. International Journal of Food Sciences and Nutrition, v. 65, n. 1, p. 9-20, 2014.

BASU, A.; LYONS, T. J. Strawberries, blueberries, and cranberries in the metabolic syndrome: Clinical perspectives. Journal of Agricultural and Food Chemistry, v. 60, n. 23, p. 5687-5692, 2011.

BEKING, K.; VIEIRA, A. An assessment of dietary flavonoid intake in The UK and Ireland. International Journal of Food Sciences and Nutrition, v. 62, n. 1, p. 17-19, 2011.

BHAGWAT, S.; HAYTOWITZ, D. B.; HOLDEN, J. M. (Org.). USDA database for the flavonoid content of selected foods, Release 3.1. Beltsville: US Department of Agriculture, Dec. 2013.

CARCACHE-BLANCO, E. J. et al. Potential cancer chemopreventive agents from $\mathrm{Arbu}$ tus Unedo. Natural Product Research, v. 20, n. 4, p. 327-334, 2006.

CASSIDY, A. et al. Habitual intake of flavonoid subclasses and incident hypertension in adults. The American Journal of Clinical Nutrition, v. 93, n. 2, p. 338-347, 2011.

CHUN, O. K.; CHUNG, S. J; SONG, W. O. Estimated dietary flavonoid intake and major food sources of U.S. The Journal of Nutrition, v. 137, n. 5, p. 1244-1252, 2007.

COPPEDÈ, F. et al. Genes and the environment in neurodegeneration. Bioscience Reports, v. 26 , n. 5 , p. $341-367,2006$.

DEFEUDIS, F. V.; DRIEU, K. Ginkgo biloba extract (EGb 761) and CNS functions basic studies and clinical applications. Current Drug Targets, v. 1, n. 1, p. 25-58, 2000.

EL MOHSEN, M. A. et al. Absorption, tissue distribution and excretion of pelargonidin 
and its metabolites following oral administration to rats. British Journal of Nutrition, London, v. 95, n. 1, p. 51-58, 2006.

ERIKSEN, J. L.; WSZOLEK, Z.; PETRUCELLI, L. Molecular pathogenesis of Parkinson disease. Archives of Neurology, v. 62 , n. 3, p. 353-357, 2005.

FAO. FOOD AND AGRICULTURE ORGANIZATION OF THE UNITED NATIONS. Food Balance Sheets: a Handbook. Roma: FAO, 2001.

FARIA, A.; MATEUS, N.; CALHAU, C. Flavonoid transport across blood-brain barrier: Implication for their direct neuroprotective actions. Nutrition and Aging, v. 1, n. 2, p. 89-97, 2012.

FIGUEIRA, I. et al. Polyphenols beyond barriers: a glimpse into the brain. Current Neuropharmacology, 2016. Full-Thematic issue on "Neuroprotection mechanisms and their potential applications" (No prelo).

FINN, C. E. Blackberries. In: HANCOCK, J. F. (Ed.) Temperate fruit crop breeding. Netherlands: Springer, 2008.

GROSSO, G. et al. Estimated dietary intake and major food sources of polyphenols in the Polish arm of the HAPIEE study. Nutrition, v. 30, n. 11, p. 1398-1403, 2014.

HERVERT-HERNANDEZ, D. et al. The contribution of fruits and vegetables to dietary intake of polyphenols and antioxidant capacity in a Mexican rural diet: Importance of fruit and vegetable variety. Food Research International, v. 44, n. 5, p. 1182-1189, 2011.

HORNYKIEWICZ, O. L-DOPA: from a biologically inactive amino acid to a successful therapeutic agent. Amino acids, v. 23, n. 1-3, p. 65-70, 2002.

ISACSON, D. et al. Fluctuating functions related to quality of life in advanced $\mathrm{Pa}$ rkinson disease: effects of duodenal levodopa infusion. Acta Neurologica Scandinavica, v. 118, n. 6 , p. $379-386,2008$.
JANKOVIC, J. Motor fluctuations and dyskinesias in Parkinson's disease: clinical manifestations. Movement Disorders, v. 20, n. S11, p. S11-S16, 2005.

JENNINGS, D. L. Raspberries and blackberries: Their breeding, diseases and growth. London: Academic Press, 1988.

JOHANNOT, L.; SOMERSET, S. M. Age-related variations in flavonoid intake and sources in the Australian population. Public Health Nutrition, v. 9, n. 08, p. 1045-1054, 2006.

KOLEHMAINEN, M. et al. Bilberries reduce lowagrade inflammation in individuals with features of metabolic syndrome. Molecular Nutrition \& Food Research, v. 56, n. 10, p. 1501-1510, 2012.

MACEDO, D. et al. (Poly) phenols protect from $\alpha$-synuclein toxicity by reducing oxidative stress and promoting autophagy. Human molecular genetics, v. 24, n. 6 , p. 1717-1732, 2015.

MALIN, D. H. et al. Short-term blueberry-enriched diet prevents and reverses object recognition memory loss in aging rats. $\mathrm{Nu}$ trition, v. 27, n. 3, p. 338-342, 2011.

MANACH, C. et al. Polyphenols: food sources and bioavailability. The American Journal of Clinical Nutrition, v. 79, n. 5, p. 727-747, 2004.

MANACH, C. et al. Bioavailability and bioefficacy of polyphenols in humans. I. review of 97 bioavailability studies. The American Journal of Clinical Nutrition, v. 81, n. 1, p. 230S-242S, 2005.

MANDEL, S.; YOUDIM, M. B. Catechin polyphenols: Neurodegeneration and neuroprotection in neurodegenerative diseases. Free Radical Biology and Medicine, v. 37, n. 3, p. 304-317, 2004.

MATTSON, M. P.; CHENG, A. Neurohormetic phytochemicals: Low-dose toxins that induce adaptive neuronal stress respon- 
ses. Trends in neurosciences, v. 29, n. 11, p. 632-639, 2006.

MECOCCI, P. et al. Nutraceuticals in cognitive impairment and Alzheimer's. In: MANCUSO, C.; GAETANI, S. (Ed.). Preclinical and clinical issues in Alzheimer's disease drug research and development. Frontiers Media SA, 2015. p. 88.

MEHTA, A. et al. Excitotoxicity: Bridge to various triggers in neurodegenerative disorders. European Journal of Pharmacology, v. 698 , n. 1, p. 6-18, 2013.

NEVEU, V. et al. Phenol-Explorer: An online comprehensive database on polyphenol contents in foods. Database, v. 2010, p. bap024, 2010.

OVASKAINEN, M. L. et al. Dietary intake and major food sources of polyphenols in Finnish adults. The Journal of Nutrition, v. 138, n. 3, p. 562-566, 2008.

PALMELA, I. M. et al. Elevated levels of bilirubin and long-term exposure impair human brain microvascular endothelial cell integrity. Current Neurovascular Research, v. 8, n. 2, p. 153-169, 2011.

PAREDES-LOPEZ, O. et al. Berries: Improving human health and healthy aging, and promoting quality life - a review. Plant foods for human nutrition, v. 65, n. 3, p. 299-308, 2010.

PEREZ-JIMENEZ, J. et al. Dietary intake of 337 polyphenols in French adults. The American Journal of Clinical Nutrition, v. 93, n. 6, p. 1220-1228, 2011.

PIMPÃO, R. C. et al. Urinary metabolite profiling identifies novel colonic metabolites and conjugates of phenolics in healthy volunteers. Molecular Nutrition \& FoodResearch, v. 58, n. 7, p. 1414-1425, 2014.

PIMPÃO, R. C. et al. Analysis of phenolic compounds in Portuguese wild and commercial berries after multienzyme hydrolysis. Journal of Agricultural and Food Chemistry, v. 61, n. 17, p. 4053-4062, 2013.
PIMPÃO, R. C. et al. Phenolic sulfates as new and highly abundant metabolites in human plasma after ingestion of a mixed berry fruit purée. British Journal of Nutrition, London, v. 113, n. 3, p. 454-463, 2015.

PINTO, P. et al. Daily polyphenol intake from fresh fruits in Portugal: contribution from berry fruits. International journal of food sciences and nutrition, v. 64, n. 8, p. 1022-1029, 2013.

RAO, S. S.; HOFMANN, L. A.; SHAKIL, A. Parkinson's disease: Diagnosis and treatment. American Family Physician, v. 74, n. $12,2006$.

SANTOS, C. N. et al. (Poli)fenóis de pequenos frutos: digestão, metabolização, biodisponibilidade e evidências de efeitos protetores em doenças neurodegenerativas, Boletim de Biotecnologia, Sociedade Portuguesa de Biotecnologia, Série 2, n. 6, p. 16-18, 2015.

SAURA-CALIXTO, F.; SERRANO, J.; GOÑI, I. Intake and bioaccessibility of total polyphenols in a whole diet. Food Chemistry, v. 101, n. 2, p. 492-501, 2007.

SCALBERT, A. et al. Dietary polyphenols and the prevention of diseases. Critical Reviews in Food Science and Nutrition, v. 45, n. 4, p. 287-306, 2005.

SCARMEAS, N. et al. Mediterranean diet and Alzheimer disease mortality. Neurology, v. 69, n. 11, p. 1084-1093, 2007.

SCARMEAS, N. et al. Mediterranean diet and mild cognitive impairment. Archives of Neurology, v. 66, n. 2, p. 216-225, 2009.

SCARMEAS, N. et al. Mediterranean diet and risk for Alzheimer's disease. Annals of Neurology, v. 59, n. 6, p. 912-921, 2006.

SCHAFFER, S.; HALLIWELL, B. Do polyphenols enter the brain and does it matter? Some theoretical and practical considerations. Genes \& Nutrition, v. 7, n. 2, p. 99-109, 2012. 
SHUKITT-HALE, B.; CHENG, V.; JOSEPH, J. A. Effects of blackberries on motor and cognitive function in aged rats. Nutritional Neuroscience, v. 12, n. 3, p. 135-140, 2009.

SHUKITT-HALE, B.; LAU, F. C.; JOSEPH, J. A. Berry fruit supplementation and the aging brain. Journal of Agricultural and Food Chemistry, v. 56, n. 3, p. 636-641, 2008.

SIMONYI, A. et al. Polyphenols In Cerebral Ischemia: Novel Targets For Neuroprotection. Molecular Neurobiology, v. 31, n. 1-3, p. 135-147, 2005.

SOUZA, J. M. et al. Dityrosine cross-linking promotes formation of stable Alpha-Synuclein polymers: Implication of nitrative and oxidative stress in the pathogenesis of neurodegenerative synucleinopathies. Journal of Biological Chemistry, v. 275, n. 24, p. 18344-18349, 2000.

SPILLANTINI, M.; GOEDERT, M. Tau and Parkinson disease. Jama, v. 286, n. 18, p. 2324-2326, 2001.

STINS, M. F.; BADGER, J.; SIK, K. K. Bacterial invasion and transcytosis in transfected human brain microvascular endothelial cells. Microbial Pathogenesis, v. 30, n. 1, p. 19-28, 2001.

TALAVÉRA, S. et al. Anthocyanin metabolism in rats and their distribution to digestive area, kidney, and brain. Journal of Agricultural and Food Chemistry, v. 53, n. 10, p. 3902-3908, 2005.

TARAZI, F. I. et al. Emerging therapies for Parkinson's disease: From bench to bedside. Pharmacology \& Therapeutics, v. 144, n. 2 , p. 123-133, 2014.

TAVARES, L. et al. Neuroprotective effect of blackberry (Rubus sp.) polyphenols is potentiated after simulated gastrointestinal digestion. Food Chemistry, v. 131, n. 4, p. 1443-1452, 2012.

TAVARES, L. et al. Neuroprotective effects of digested polyphenols from wild blackberry species. European Journal of Nutrition, v. 52, n. 1, p. 225-236, 2013.

TOUVIER, M. et al. Dual association between polyphenol intake and breast cancer risk according to alcohol consumption level: a prospective cohort study. Breast Cancer Research and Treatment, v. 137, n. 1, p. 225-236, 2013.

TRESSERRA-RIMBAU, A. et al. Dietary intake and major food sources of polyphenols in a Spanish population at high cardiovascular risk: The PREDIMED study. Nutrition, Metabolism and Cardiovascular Diseases, v. 23, n. 10, p. 953-959, 2013.

VAN DER GREEF, J.; STROOBANT, P.; VAN DER HEIJDEN, R. The role of analytical sciences in medical systems biology. Current Opinion in Chemical Biology, v. 8, n. 5, p.559-565, 2004.

VON CAMPENHAUSEN, S. et al. Prevalence and incidence of Parkinson's disease in Europe. European Neuropsychopharmacology, v. 15, n. 4, p. 473-490, 2005.

WHO. World Health Organization. Global Burden of Disease: 2004 update. 2008.

WHO. World Health Organization. Active ageing: a policy framework. Madrid: WHO, 2002.

WILLIAMSON, G.; MANACH, C. Bioavailability and bioefficacy of polyphenols in humans. I. Review of 97 bioavailability studies. The American Journal of Clinical Nutrition, v. 81, n. 1, p. 230S-242S, 2005.

WIMO, A. et al. The economic impact of dementia in Europe in 2008-cost estimates from the Eurocode project. International Journal of Geriatric Psychiatry, v. 26, n. 8, p. 825-832, 2011.

ZAMORA-ROS, R. et al. Estimation of dietary sources and flavonoid intake in a Spanish adult population (EPIC-Spain). Journal of the American Dietetic Association, v. 110, n. 3, p. 390-398, 2010. 